\title{
Study of growth matter index and cosmic implications in dynamical Chern-Simons modified gravity
}

\author{
Abdul Jawad $^{\mathrm{a}}$, Shamaila Rani ${ }^{\mathrm{b}}$, Saba Qummer ${ }^{\mathrm{c}}$, Amir Sharif ${ }^{\mathrm{d}}$ \\ Department of Mathematics, COMSATS University Islamabad, Lahore Campus, Islamabad 54000, Pakistan
}

Received: 12 February 2021 / Accepted: 25 July 2021 / Published online: 10 August 2021

(C) The Author(s) 2021

\begin{abstract}
In the framework of dynamical Chern-Simons theory of gravity, we study the recent cosmic expansion with acceleration of the universe. We take interacting scenario of dark energy and dark matter with three cutoffs like Granda-Oliveros, higher order derivative of Hubble parameter and generalized holographic dark energy model. In the flat Friedmann-Robertson-Walker universe, well-known cosmological parameters have been calculated. We study the perturbation of matter density growth, growth factor and growth index behavior for the underlying framework. The cosmological parameters like equation of state parameter, deceleration parameter, and stability of each model are discussed. The outcomes of these models represent the cosmic expansion of the universe with acceleration.
\end{abstract}

\section{Introduction}

Cosmic microwave background radiation (CMB) data [1-3], galaxy redshift surveys [4], gravitational lens surveys [5], astrophysical measurements of type Ia supernovae $[6,7]$ and large scale structure credibly prove an accelerated evolution of the observable cosmos. In this regard, different cosmological phenomena [8] has been made for observations. A force called dark energy (DE) is suggested to be responsible for the evolution but its existence is very mysterious. A lot of attempts have been made through DE models to investigate its existence as well as certain popular modified gravities. Numerous observations have also been carried out to measure cosmic acceleration from different factors $[9,10]$.

Cosmic acceleration has also been studied by a compelling modified gravities that has appeared i.e modified Chern-

\footnotetext{
a e-mails: jawadab181@yahoo.com ; abduljawad@cuilahore.edu.pk

b e-mails: shamailatoor.math@yahoo.com ;

drshamaila-rani@cuilahore.edu.pk (corresponding author)

c e-mail: sabaqummer143@gmail.com

de-mail: amirjutt5088@gmail.com
}

Simons (CS) model of gravity [11]. An anomaly-cancelling correction to the Einstein-Hilbert action involves the limit of string theory, deriving the modified gravity of CS as an appropriate theory. Violation of parity symmetry was investigated by using this scenario [11], which occurs in all 4D compactifications of the theory of perturbative strings and more in loop quantum gravity as in well, when a scalar field emulates a Barbero-Immirzi parameter, coupled with the invariant Nieh-Yan [12-15]. The Pontryagin term is well recognized not to impact the field equations in the modified gravity of CS and hence the so-called CS correction consists of the Pontryagin density processed by the scalar field and it can be viewed either as a non-dynamic field of history or as a dynamic field of evolution. Therefore, the dynamic CS modified gravity is considered $[16,17]$ in the latter case. Some attempts have provided the limits to the CS parameters [18].

Out of all the cosmological models, $\Lambda \mathrm{CDM}$ model has most compatibility with observational schemes. But, $\Lambda \mathrm{CDM}$ still suffer some problems i.e fine tuning and coincidence problem. To address such problems dynamical dark energy models have been proposed such i.e Chaplygin gas, holographic dark energy (HDE), quintessence, K-essence and ghost [19]. The HDE is an interesting DE model which is proposed in quantum gravity scenario with the help of holographic principle [20]. The HDE provides a relationship between the density of the quantum field to the vacuum, UV and IR cutoffs. In order to examine the HDE the entropy of the black hole plays an important role. Cohen et al. [21] proposed that the total energy of the system with size $L$ should not exceed the mass of black hole. The energy density of HDE model is defined as

$\rho=3 c^{2} M_{p}^{2} L^{-2}$,

where $c, M_{p}$ and $L$ are some numerical constant, reduce Planck mass and IR cutoff respectively. To study the cosmic expansion, different IR cutoffs such as Hubble radius, event horizon, GO length, Ricci length and the age of universe etc. 
The particle horizon is suggested for HDE density as an alternative scale or IR cut-off but the EoS parameter for this model remains $\geq 1 / 3$ [22], which does not help to describe the universe's existing scenario. Li [23] has suggested the cosmic event horizon as a characteristics length in order to eliminate this conspiracy, which explains the present acceleration of the universe $[24,25]$. However as an IR cut-off, the HDE with a event horizon is plagued with some conceptual issues. The causality question pointed out by Cai [26] is an interesting limitation of this model, which is described as, why should a global quantity be calculated by a local quantity? The DE behave like local quantity while event horizon is treated as global quantity in space time.

Ricci DE (RDE) [27] and its generalized form [28,29] have been proposed to resolve this problem. Cai et al. [30] described holographic RDE (HRDE) relationship with the explanatory interaction scale i.e., for the flat universe $R^{-2}=\operatorname{Max}\left(2 H^{2}+\dot{H},-\dot{H}^{2}\right)$. They found only the models which are associated with the collected data is $R^{-2}=$ $\operatorname{Max}\left(2 H^{2}+\dot{H}\right)$ as an IR cut-off coherent to the recent observational scheme. It has been suggested that either $\dot{H}$ or $H^{2}$ does not assist to explain the rapid growth of the universe late in time, but better outcome can be get through their combination [26]. As a consequence of the proposed generalized RDE (GRDE) and generalized HDE (GHDE) models, various cosmological schemes have been tested their evolution and viability [31,32]. Nojiri and Odinstov [33] investigate GHDE and its generalized theory. Many authors [34,35] have presented the dynamical scalar fields and relevant potentials in the form of several DE models. In particular HDE models with Hubble, future event and Granda-Oliveros (GO) IR cutoff in the models of flat and non-flat universe. Interestingly, scalar field effects and their potential have been established that provides a useful description of the cosmic expansion. The expansion and stability of HDE interaction with GO IR cut-off in the non-flat universe is explored in a recent paper by Sharif and Jawad [36]. The evolution and stable behavior of the interaction of GHDE energy density with cold dark matter (CDM), $L_{G O}$ cut-off and higher order derivatives of the Hubble parameter in the flat universe are explored. Sharif and Jawad [37] also investigated interacting GRDE and found interesting results. Some authors [38-54] have investigated various types of DE models in different scenarios and got fascinating outcomes. The study of thermodynamics in modified gravities have been studied in [55-61]. Recently, thermodynamic consequences of gravity theory have been studied in [62]).

In order to differentiate between scalar field DE and modified gravity evaluation of linear growth of matter fluctuations can be used. However, the evaluation of growth index is considered to be one of the main target and it is used to test general relativity on extra galactic scale. In literature, different cosmological models such as scalar field, $f(T), f(R)$,
Finsler-Randers and HDE are studied to evaluate analytically the functional form of growth index. In the preceptive of large scale structure, the distribution of extragalactic using various mass tracers provides principal constraints on structure formation theories. In this article, we have studied three different cosmological models i.e GO HDE model, higher order derivative of Hubble parameter model and generalized HDE model in the framework of dynamical CS modified gravity to evaluate the cosmic parameters and matter density fluctuations.

In this manuscript, we achieve the well behaved and feasible solutions of different cosmological models in the background of dynamical CS modified theory of gravity. In Sect. 2, we define the Friedmann equation of CS modified gravity for flat universe and explain the brief review of DE models. In Sect. 3, we evaluate the growth factor and growth index and also provide the graphical analysis of these parameters. Section 4 is devoted to study the cosmological parameters and evaluated the analytical and graphical solutions of these parameters in flat universe. Last section is devoted to conclude the results.

\section{Dark energy models}

In this section, we will provide a brief overview of the CS modified gravity and DE models. We consider the HDE with GO cutoff, higher order derivatives of the Hubble parameter $H$ and GHDE model.

\subsection{Dynamical Chern-Simons modified gravity}

The action of the CS modified theory is defined as [63]

$$
\begin{aligned}
S= & \frac{1}{16 \pi G} \int_{v} d^{4} x\left[\sqrt{-g} R+\frac{l}{4} \dot{\theta} R^{\rho \sigma \mu \nu} R_{\rho \sigma \mu \nu}+L_{1}\right] \\
& +S_{\text {mat }} . \\
L_{1}= & -\frac{1}{2} g^{\mu \nu} \nabla_{\mu} \theta \nabla_{\nu} \theta+V(\theta),
\end{aligned}
$$

where $\nabla_{\mu}, \theta$ and $l$ represents covariant derivative, dynamical variable and coupling constant respectively, $R^{\rho \sigma \mu \nu} R_{\rho \sigma \mu \nu}$ is a Pontryagin term, potential term is denoted by $V(\theta)$ and assumed to be zero for simplicity and $S_{m a t}$ corresponds to the matter action. In various field equations of CS theory, the variance of the action w.r.t $g_{\mu \nu}$ metric tensor and $\theta$ represents the scalar field that frequently occurs.

$$
\begin{aligned}
G_{\mu \nu}+l C_{\mu \nu} & =8 \pi G T_{\mu \nu}, \\
g^{\mu \nu} \nabla_{\mu} \nabla_{\nu} \theta & =\frac{-l}{64 \pi} R^{\rho \sigma \mu \nu} R_{\rho \sigma \mu \nu},
\end{aligned}
$$


where, $G_{\mu \nu}$ and $C_{\mu \nu}$ are the Einstein and Cotton tensor. The Cotton tensor is given as follows

$C_{\mu \nu}=\frac{-1}{2 \sqrt{-} g}\left(\left(\nabla_{\rho} \theta\right) \varepsilon^{\rho \beta \mathcal{T}\left(\mu \nabla_{\mathcal{T}} R_{\beta}^{\nu}\right)}\right)+\left(\nabla_{\sigma \nabla_{\rho} \theta}\right) \times R^{\rho(\mu \nu) \sigma}$.

In general, $T_{\mu \nu}$ consists of matter $\left(T_{\mu \nu}^{m}\right)$ and scalar $\left(T_{\mu \nu}^{\theta}\right)$ parts defined as

$T_{\mu \nu}^{\theta}=\nabla_{\mu} \theta \nabla_{\nu} \theta-\frac{1}{2} g_{\mu \nu} \nabla^{\rho} \theta \nabla_{\rho} \theta$

$T_{\mu \nu}^{m}=(\rho+P) u_{\mu} u_{\nu}+p g_{\mu \nu}$.

Here $u_{\mu}$ is also the four-vector velocity in the space-time co-moving coordinates.

\subsection{Model 1: GO-HDE model}

In literature, in the view of length scale $L$ different HDE models have been proposed. horizion is an alternate choice

- The Hubble length $L$ is a an easiest choice [64,65], i.e $L=H^{-1}$. In this scenario the DE density will be very closer to the observational scheme, but the present day cosmic evolution cannot be recovered.

- The particle horizon is another option, in this framework HDE model is unable to provide an cosmic expansion.

- The future event is an alternate choice for the scale length [23]. This HDE model is best fit with the late time expansion, The choice of future event horizon solve coincidence and fine tuning problems $[66,67]$.

- The curvature of spacetime is also a simple choice for $L$ is, for which Ricci HDE model shows compatibility with the supernova type Ia $[68,69]$.

- Granda and Oliveros (GO) provides a scale length depending on the combination of Hubble parameter and its derivative w.r.t time. In this regard, late time phase of the universe is recovered and model of DE is consistent with Ia supernova observations [70]. It has been shown that HDE models depending upon GO cutoff are best fit to observational growth rate data in perturbation level as concordance model [71]. Thus, GO scale length given by $[72,73]$, is known as this new IR cut-off $L_{G O}$

$$
L_{G O}=\left(\alpha \dot{H}+\beta H^{2}\right)^{\frac{-1}{2}}
$$

where, $\alpha$ and $\beta$ are constant in above equation.

As the fundamental basis of the HDE model is omitted the HDE energy density expression with a cutoff of $L_{G O}$ is given by

$\rho_{\theta 1}=3 c^{2}\left(\alpha \dot{H}+\beta H^{2}\right)$.
We have $c=0.818_{-0.097}^{+0.113}$ for a flat universe $[74,75]$. It is fairly evident that the Planck mass $M_{p}$ is supposed to be converted to unity. In the limiting case, corresponding to $\alpha=1$ and $\beta=2$, the $L_{G O}$ decreases to Ricci's average scale curvature radius. In [76], the authors discussed some cosmological parameters by taking into account GO cutoff in dynamical Chern-Simons theory. They considered $c=1$ to draw the cosmic behavior of parameters.

2.3 Model II: higher order derivative of hubble parameter model

In this work we'll consider the DE model suggested in [77] having three distinct terms: one is proportional to the squared Hubble parameter, second term is proportional to the first derivative of $H$ w.r.t cosmic time and last term is proportional to the second order derivative of $H$ w.r.t time

$\rho_{\theta 2}=3\left(\alpha \frac{\ddot{H}}{H}+\beta \dot{H}+\zeta H^{2}\right)$,

where $\alpha, \beta$, and $\zeta$, are positive constant parameters, such as [78]. The first term is divided by $H$ in order that all the three terms have equal dimensions. The energy density given in Eq. (8) is considered as generalization of Ricci DE (RDE) model and the DE energy density with GO cut-off.

The profile of DE and expansion of universe depends on $\alpha, \beta$ and $\zeta$ also the primary motivation for this model is to reduce the age factor of three different objects, namely APM $08279+5255$ and LBDS 53 W091 [79-81] for the specific choice of the parameters. A special case for $\alpha=0$, we can get the energy density of the DE with GO cutoff and in the other case can be obtained for $\alpha=0, \beta=1$ and $\zeta=2$, the $\mathrm{RDE}$ model for a flat universe is recovered.

\subsection{Model III: GHDE model}

The GHDE model is defined as [31]

$\rho_{\theta 3}=3 c^{2} H^{2} f\left(\frac{R}{H}\right)$,

where, $c$ is a constant, $R$ is the Ricci scalar and $f\left(\frac{R}{H^{2}}\right)$ is the positive function of dimensionless variables. Further, with the help of Hubble horizon, we can reconstruct the existing holographic as an IR cut off for $f\left(\frac{R}{H^{2}}\right)=\frac{R}{H^{2}}$. This function can be written in linear combination form as

$f\left(\frac{R}{H^{2}}\right)=1+\alpha\left(\frac{R}{H^{2}}-1\right)$,

where, $\alpha$ is the constant parameter. Note that for $\alpha=0$ we obtain HDE while, RDE can be recovered for $\alpha=1$. Furthermore, for the parameterization of $\alpha$, GRDE and GHDE gives the equivalent results. 


\section{Field equations}

The FRW metric is given by

$d s^{2}=-d t^{2}+\frac{a^{2}(t) d r^{2}}{1-k r^{2}}+r^{2} a^{2}(t)\left(d \theta^{2}+\sin ^{2} \theta d \phi^{2}\right)$,

where $a(t)$ is the scale factor and $k$ is the spatial curvature represented the universe as open for $k=-1$, flat for $k=0$ and closed for $k=1$. In the framework of CS gravity, for flat FRW universe, the first Friedmann equation can be obtained by using Eqs. (3) and (5) as follows

$H^{2}=\frac{1}{3}\left(\rho_{m}+\rho_{\theta j}\right)+\frac{1}{6} \dot{\theta}^{2}$.

Here, the dot corresponds to the derivative of $a(t)$ w.r.t cosmic time and $M_{p}^{-1}=8 \pi G=1$, also, $8 \pi G$ is constant term and $8 \pi$ is required so that GR reduces to Newtonian gravity in the appropriate limit. The FRW metric provides that $R^{\rho \sigma \mu \nu} R_{\rho \sigma \mu \nu}=0$, hence the field equation (2) is related with the scalar field takes the following form

$g^{\mu \nu} \nabla_{\mu} \nabla_{\nu} \theta=g^{\mu \nu}\left[\partial_{\nu} \partial_{\mu} \theta\right]=0$.

With the assumption $\theta=\theta(t)$, the above equation leads to

$\ddot{\theta}+3 H \dot{\theta}=0$,

which implies that $\dot{\theta}=D a^{-3}, c$ is a constant of integration. Thus, we can get the expression for Eq. (12) as

$H^{2}=\frac{1}{3}\left(\rho_{m}+\rho_{\theta j}\right)+\frac{1}{6}\left(D^{2} a^{-6}\right)$.

Leading Eq. (15) to

$1=\Omega_{m}+\Omega_{\theta j}+\Omega_{c s}$.

here,

$\Omega_{m}=\frac{\rho_{m}}{3 H^{2}}, \quad \Omega_{\theta j}=\frac{\rho_{\theta j}}{3 H^{2}}, \quad \Omega_{c s}=\frac{D^{2}}{6 H^{2} a^{6}}$.

For the standard model of evolution of the universe it is considered that DE and DM are the dominant sources. These ingredients have been discovered from their gravitational effects and have unavoidable degeneracy. As the universe possess the specific ratio of $\mathrm{DE}$, so its look natural to look into the interaction between other components of the universe. Because of this, the interacting scenario between DE and DM have attained a lot of attentions. It is considered that the interaction of DE and DM influence the perturbation dynamics and effects the lowest multipoles of CMB spectrum. Also, data of SNIa, CMB and LSS also inferred the interaction of DE and DM during the expansion of the universe. Furthermore, interaction between DE and DM provides a plentiful cosmological dynamics as in comparison to the noninteracting models. We assume the interaction between DE and CDM which leads to following non-conservation equations

$$
\begin{aligned}
\dot{\rho}_{m}+3 H \rho_{m} & =Q, \\
\dot{\rho}_{j}+3 H\left(\rho_{\theta j}+p_{j}\right) & =-Q .
\end{aligned}
$$

Here, $Q$ shows the rate of energy exchange between two constituents of the universe i.e DE and DM, whose different forms available in the literature. If $Q>0$, it shows that energy is being transferred from DE to DM. For $Q<0$, the energy is being transferred DM to DE. In literature, the usual choice of $Q$ comprises the linear terms of either dark matter, dark energy or a linear combination of both. In our work, we assume the following form

$Q=3 d^{2} H \rho_{m}$,

with $d^{2}$ appears as interacting constant. In view of this interaction term, Eq. (18) gives us CDM density as

$\rho_{m}=\rho_{m_{0}}(1+z)^{3\left(1-d^{2}\right)}$,

where $x=\ln a$ and $\rho_{m_{0}}$ is an integration constant.

\section{For Model 1}

Using Eqs. ((7), (21)) in (15), we get the following equation

$$
\begin{aligned}
& \frac{d E^{2}}{d x}-\frac{2}{c^{2} \alpha}\left(1-c^{2} \beta\right) E^{2} \\
& =\frac{-2}{c^{2} \alpha}\left(\Omega_{m_{0}}(1+z)^{3\left(1-d^{2}\right)}+\Omega_{c s_{0}}(1+z)^{6}\right)
\end{aligned}
$$

where $E^{2}=\frac{H^{2}}{H_{0}^{2}}$ with present value of the parameter $H_{0}$ and the general solution of Eq. (22) is given as

$$
\begin{aligned}
E^{2}= & b(1+z)^{\frac{2}{c^{2} \alpha}\left(c^{2} \beta-1\right)}+\frac{2 \Omega_{m_{0}}(1+z)^{3\left(1-d^{2}\right)}}{3 c^{2} \alpha\left(1-d^{2}\right)-2\left(c^{2} \beta-1\right)} \\
& +\frac{2 \times \Omega_{c s_{0}}(1+z)^{6}}{6 c^{2} \alpha-2\left(c^{2} \beta-1\right)} .
\end{aligned}
$$

Where $b$ occurs as integration constant which can be calculated by utilizing initial condition $a(t=0)=1$ as

$b=\frac{-2}{c^{2} \alpha}\left[\frac{\Omega_{m_{0}}}{3\left(1-d^{2}\right)-\frac{2}{c^{2} \alpha}\left(c^{2} \beta-1\right)}+\frac{\Omega_{c s_{0}}}{6-\frac{2}{c^{2} \alpha}\left(c^{2} \beta-1\right)}\right]$.

For Model 2

Inserting Eqs. ((8), (21)) in (15), one can get

$$
\begin{aligned}
& \frac{d^{2} E^{2}}{d x^{2}}+\frac{\beta}{\alpha} \frac{d E^{2}}{d x}-\frac{2}{\alpha}(1-\zeta) E^{2} \\
& =\frac{-2}{\alpha}\left(\Omega_{m_{0}}(1+z)^{3\left(1-d^{2}\right)}+\Omega_{c s_{0}}(1+z)^{6}\right)
\end{aligned}
$$


where the general solution of the Eq. (25) is given below as

$$
\begin{aligned}
E^{2}= & \frac{b_{1}}{1+z} e^{\frac{-1}{2 \alpha}\left(\beta+\sqrt{\beta^{2}+8 \alpha-8 \alpha \zeta}\right)} \\
& +\frac{b_{2}}{1+z} e^{\frac{-1}{2 \alpha}\left(\beta-\sqrt{\beta^{2}+8 \alpha-8 \alpha \zeta}\right)} \\
& -\frac{2 \Omega_{c s_{0}}(1+z)^{6}}{36 \alpha-6 \beta+2 \zeta-2} \\
& -\frac{2 \Omega_{m_{0}}(1+z)^{3\left(1-d^{2}\right)}}{9 \alpha d^{4}+3 \beta d^{2}-18 \alpha d^{2}+9 \alpha-3 \beta+2 \zeta-2},
\end{aligned}
$$

where, $b_{1}$ and $b_{2}$ appear as integration constants. One can get these constants through initial conditions $a(t=0)=1$ and $\left.\frac{d E^{2}}{d x}\right|_{t=0}=0$ as

$$
\begin{aligned}
b_{1}= & \frac{1}{\sqrt{\beta^{2}+8 \alpha-8 \alpha \zeta}} \\
& \times\left[\frac{\left(6 \alpha\left(1-d^{2}\right)-\beta+\sqrt{\beta^{2}+8 \alpha-8 \alpha \zeta}\right) \Omega_{m_{0}}}{9 \alpha d^{4}+3 \beta d^{2}-18 \alpha d^{2}+9 \alpha-3 \beta+2 \zeta-2}\right. \\
& \left.+\frac{\left(12 \alpha-\beta+\sqrt{\beta^{2}+8 \alpha-8 \alpha \zeta}\right) \Omega_{c s_{0}}}{36 \alpha-6 \beta+2 \zeta-2}\right] \\
b_{2}= & \frac{-1}{\sqrt{\beta^{2}+8 \alpha-8 \alpha \zeta}} \\
& \times\left[\frac{\left(6 \alpha\left(1-d^{2}\right)-\beta-\sqrt{\beta^{2}+8 \alpha-8 \alpha \zeta}\right) \Omega_{m_{0}}}{9 \alpha d^{4}+3 \beta d^{2}-18 \alpha d^{2}+9 \alpha-3 \beta+2 \zeta-2}\right. \\
& \left.+\frac{\left(12 \alpha-\beta-\sqrt{\beta^{2}+8 \alpha-8 \alpha \zeta}\right) \Omega_{c s_{0}}}{36 \alpha-6 \beta+2 \zeta-2}\right] .
\end{aligned}
$$

For Model 3:

For this model, we will utilize the following relations for flat universe $(k=0)$

$$
\frac{R}{H^{2}}=\frac{\dot{H}+2 H^{2}}{H^{2}}=2+\frac{1}{2 E^{2}} \frac{d E^{2}}{d x},
$$

Using Eqs. (9), (10), (21), (29) and (15), we obtain

$$
\begin{aligned}
& \frac{d E^{2}}{d x}-\frac{2 E^{2}}{c^{2} \alpha}\left(1-c^{2}-c^{2} \alpha\right) \\
& =\frac{-2}{c^{2} \alpha}\left(\Omega_{m_{0}}(1+z)^{3\left(1-d^{2}\right)}+\Omega_{c s_{0}}(1+z)^{6}\right) .
\end{aligned}
$$

The general solution of Eq. (30) leads to

$$
\begin{aligned}
E^{2}= & \frac{b_{4}}{1+z} \times e^{\frac{-2}{c^{2} \alpha}\left(c^{2} \alpha+c^{2}-1\right)} \\
& +\frac{2 \times \Omega_{m_{0}} \times(1+z)^{-3 c^{2} \alpha\left(1-d^{2}\right)}}{3 c^{2} \alpha\left(1-d^{2}\right)-2\left(c^{2} \alpha+c^{2}-1\right)} \\
& +\frac{2 \times \Omega_{c s_{0}} \times(1+z)^{6}}{6 c^{2} \alpha-2\left(c^{2} \alpha+c^{2}-1\right)},
\end{aligned}
$$

where $b_{4}$ appears as integration constant. By using initial condition $a(t=0)=1$, we get

$$
\begin{aligned}
b_{4}= & \frac{-2 \times \Omega_{m_{0}}}{3 c^{2} \alpha\left(1-d^{2}\right)-2\left(c^{2} \alpha+c^{2}-1\right)} \\
& -\frac{2 \times \Omega_{c s_{0}}}{6 c^{2} \alpha-2\left(c^{2} \alpha+c^{2}-1\right)} .
\end{aligned}
$$

\section{Growth of matter density perturbation}

From last few years, it is completely understandable that the study of the cosmic dynamics is insufficient to differentiate between modified gravities and the standard model of cosmology. A possible way to check the deviations and to smash the degeneracy between the alternative theories is to study the density perturbations over the homogeneous and isotropic framework [82-84]. The growth rate of LSS in GR can be calculated through matter density perturbation in linear system which provides the following differential equation $\ddot{\delta}_{m}+2 H \dot{\delta}_{m}-4 \pi G \rho_{m} \delta_{m}=0$.

The solution of above differential equation is $\delta_{m}(t) \propto D(t)$, with $D(t)$ denoting the linear growing mode. We use the following growth factor to differentiate different models with matter perturbation

$g \equiv \frac{d \ln \delta_{m}}{d x}$.

Using Eq. (33) in (34), we have

$\frac{d g}{d x}+g^{2}+\left(\frac{1}{2 E^{2}} \frac{d E^{2}}{d x}+2\right) g=\frac{3}{2} \Omega_{m_{0}} E^{-2}(1+z)^{3\left(1-d^{2}\right)}$.

It is mentioned here that above equation cannot be solved analytically. Thus, we solve it numerically for growth factor $g$ by inserting the expression of $E^{2}$ for three different models of DE. It is remarkable to note that the solution of above equation can can be approximated in [85-88] known as growth factor $g$

$g=\Omega_{m}^{\gamma}$.

This is well known approximation for growth factor. Here $\gamma$ denotes the growth index parameter and has different approximated observational values for matter dominated eras, e.g., $\frac{2}{3}$ [87] and $\frac{4}{7}$ [88]. More generally, the growth index may not be a constant but rather evolve with redshift. However, its suggested observational range is $0<\gamma<1$ in the literature. In order to check the behavior of $g$ and $\gamma$, we use following values of constants. For model 1, 2 and 3 we take $c=1.2, d=0.7, d=0.8, d=0.9, \Omega_{m_{0}}=0.27$ and $\Omega_{c s_{0}}=0.05$ as common constants. But for model 1, we use $\alpha=0.5$ and $\beta=1$, for model 2 we have $\alpha=-0.5, \beta=1$ and $\zeta=0.5$ and for model three we use the $\alpha=0.5$. 

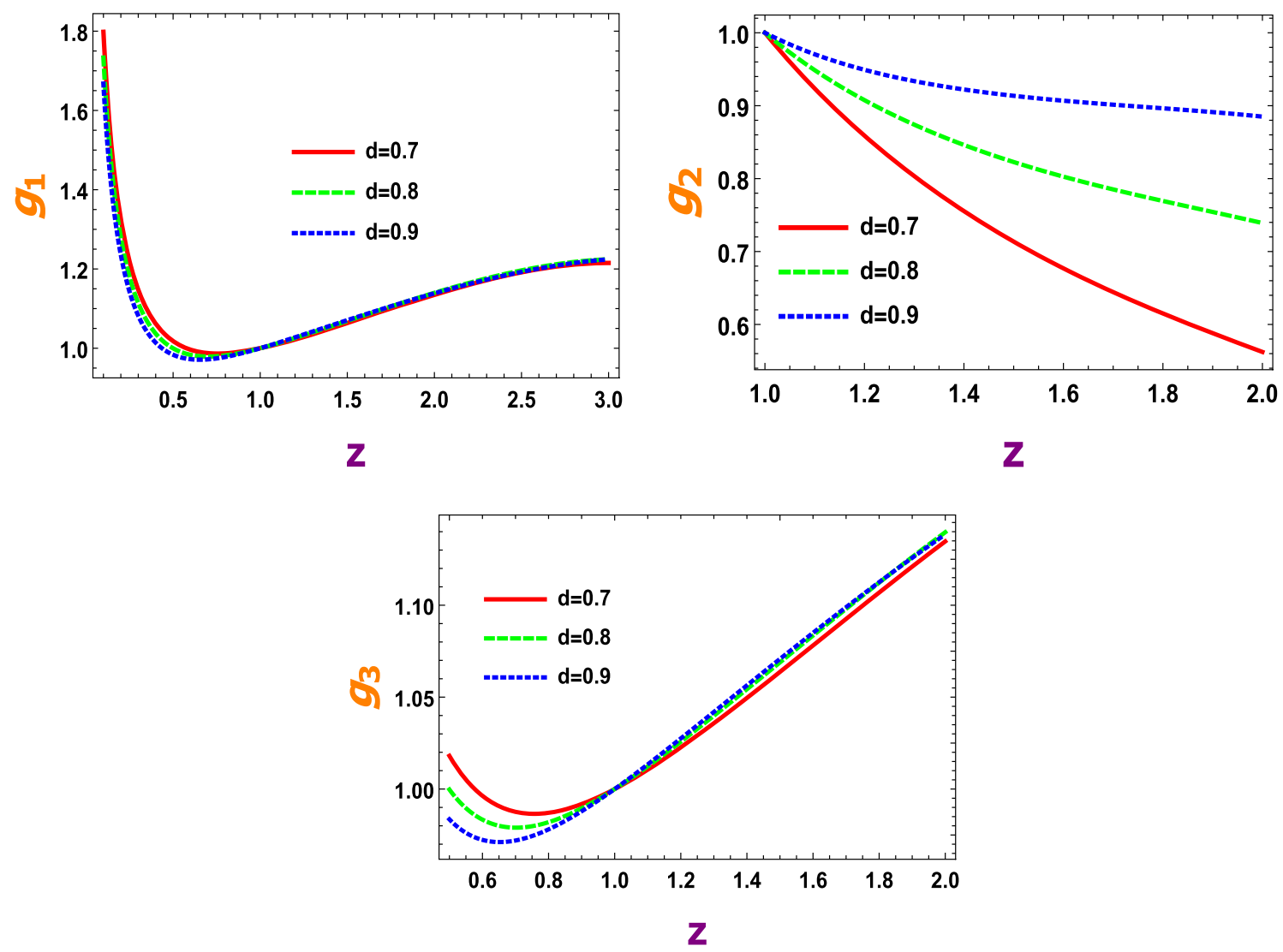

Fig. 1 Plots of $g_{1}, g_{2}$ and $g_{3}$ versus $z$

- Growth factor: We draw the plots of Eq. (43) in terms of redshift parameter numerically for three different models $(1,2,3)$ of HDE, respectively as shown in Fig. 1 . We observe that this growth factor shows positive behavior with respect to redshift parameter and lies within observational suggested ranges [89] (references therein) for all three models of HDE.

- Growth index parameter: Numerical evaluation of this parameter against redshift parameter is done in Fig. 2, for three models respectively. In these plots, the values of constants remain same as utilized in growth factor graphs. We attain the $0<\gamma<1$ for all three models, compatible behavior with the works [87-89] and different behavior from $\Lambda$ CDM model. We discuss the dependence of the growth index $\gamma$ on the dimensionless matter energy density For $\Lambda$ CDM Gong [90] found the $\Lambda$ CDM limit for $\gamma=0.64_{-0.15}^{+0.17}$ and our results are different from $\Lambda \mathrm{CDM}$ model.Furthermore, the plot of $\gamma$ against redsift for model 2 is shown in the right panel of Fig. 2. To distinguish our results from $\Lambda \mathrm{CDM}$ model we have plotted points on red trajectory which shows the $\Lambda \mathrm{CDM}$ limit for $d=0.7$. Other 2 models does not show any correspondence with $\Lambda \mathrm{CDM}$.

\section{Cosmological parameters}

In this section, we provide some cosmological parameters such as equation of state parameter, deceleration parameter and squared speed of sound.

\subsection{Equation of state parameter}

We also calculate the EoS parameter for three different HDE models by using

$\omega_{\theta j}=\frac{p_{j}}{\rho_{\theta j}}$,

where $\rho$ and $p$ denotes the density and pressure respectively also $j$ varies for three different DE models. If $j=1$ then it shows GO-HDE model, for $j=2$ represents the model of higher order derivative of the hubble parameter and $j=3$ is for GHDE and discuss the expansion of the universe. For first model of HDE (with GO cutoff), we can calculate EoS parameter by using Eqs. (19)-(21) and (23) as

$$
\begin{aligned}
\omega_{\theta 1}= & -\left[\frac{1}{3}\left(\frac{-2}{c^{2} \alpha}\left(c^{2} \beta-1\right) x\right)\left(\frac{-2 \beta}{\alpha}+\frac{2}{c^{2} \alpha}+3\right)\right. \\
& b(1+z)^{\frac{2}{c^{2} \alpha}}\left(c^{2} \beta-1\right)-3\left(1-d^{2}\right)
\end{aligned}
$$



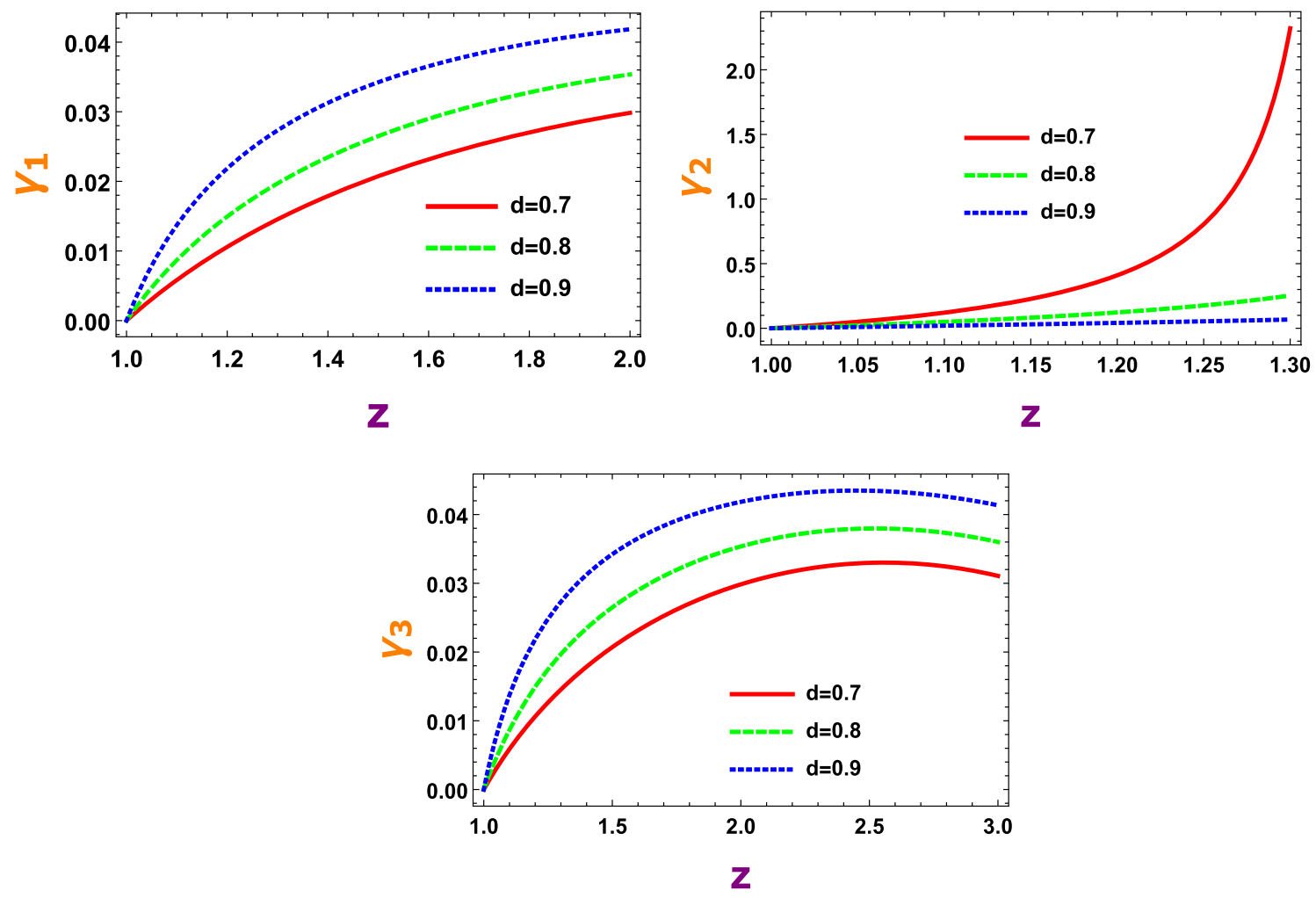

Fig. 2 Plots of growth index $\gamma_{1}, \gamma_{2}$ and $\gamma_{3}$ against $z$

$$
\begin{aligned}
& \times \frac{2 d^{2} \Omega_{m_{0}}(1+z)^{3\left(1-d^{2}\right)}}{3 c^{2} \alpha-3 \alpha c^{2} d^{2}-2 \beta c^{2}+2} \\
& \left.+\frac{6 c^{2}(\beta-3 \alpha) \Omega_{c s_{0}}(1+z)^{6}}{3 c^{2} \alpha-c^{2} \beta+1}\right]\left[\frac{-2}{c^{2} \alpha}\left(c^{2} \beta-1\right)\right. \\
& \times b(1+z) \frac{2}{c^{2} \alpha}\left(c^{2} \beta-1\right) \\
& -3\left(1-d^{2}\right) \frac{c^{2}\left(2 \beta-3 \alpha+3 \alpha d^{2}\right) \Omega_{m_{0}}(1+z)^{3\left(1-d^{2}\right)}}{3 c^{2} \alpha-3 \alpha c^{2} d^{2}-2 \beta c^{2}+2} \\
& \left.-\frac{6 c^{2}(\beta-3 \alpha) \Omega_{c s_{0}}}{3 c^{2} \alpha-c^{2} \beta+1}\right]^{-1}
\end{aligned}
$$

For Model 2 (HDE with higher order derivatives of $H$ ), EoS parameter can be acquired with the help Eqs. (19)-(21) and (26) as

$$
\begin{aligned}
\omega_{\theta 2}= & -\left[\frac{6 \alpha-\beta-\sqrt{\beta^{2}+8 \alpha-8 \alpha \zeta}}{6 \alpha}\right. \\
& \frac{b_{1}}{1+z} e^{\frac{-1}{2 \alpha}\left(\beta+\sqrt{\beta^{2}+8 \alpha-8 \alpha \zeta}\right)}+(6 \alpha)^{-1} \\
& \times\left(6 \alpha-\beta+\sqrt{\beta^{2}+8 \alpha-8 \alpha \zeta}\right) \\
& \frac{b_{2}}{1+z} e^{\frac{-1}{2 \alpha}\left(\beta-\sqrt{\beta^{2}+8 \alpha-8 \alpha \zeta}\right)} \\
& -\left(2 d^{2} \Omega_{m_{0}}(1+z)^{3\left(1-d^{2}\right)}\right)
\end{aligned}
$$

$$
\begin{aligned}
& \times\left(9 \alpha d^{4}+3 \beta d^{2}-18 \alpha d^{2}+9 \alpha-3 \beta+2 \zeta-2\right)^{-1} \\
& +\frac{(36 \alpha-6 \beta+2 \zeta)}{36 \alpha-6 \beta+2 \zeta-2} \Omega_{c s_{0}} \\
& \left.\times(1+z)^{6}\right]\left[\frac{b_{1}}{1+z} e^{\frac{-1}{2 \alpha}\left(\beta+\sqrt{\beta^{2}+8 \alpha-8 \alpha \zeta}\right)}\right. \\
& +b_{2} e^{\frac{-1}{2 \alpha}\left(\beta-\sqrt{\beta^{2}+8 \alpha-8 \alpha \zeta}\right)}-\Omega_{m_{0}}(1+z)^{3\left(1-d^{2}\right)} \\
& \times\left(9 \alpha d^{4}+3 \beta d^{2}-18 \alpha d^{2}+9 \alpha-3 \beta+2 \zeta\right) \\
& \left(9 \alpha d^{4}+3 \beta d^{2}-18 \alpha d^{2}+9 \alpha-3 \beta+2 \zeta-2\right)^{-1} \\
& \left.-\frac{(36 \alpha-6 \beta+2 \zeta) \Omega_{c s_{0}}}{36 \alpha-6 \beta+2 \zeta-2}(1+z)^{6}\right]^{-1} \text {. }
\end{aligned}
$$

For Model 3 (HDE with Ricci scalar cutoff), EoS parameter can be acquired with the help Eqs. (19)-(21) and (29)-(31) as

$$
\begin{aligned}
\omega_{\theta 3}= & -\left[\frac{1}{3}\left(\frac{2}{c^{2} \alpha}+1-\frac{2}{\alpha}\right) \frac{b}{1+z} e^{\frac{-2}{c^{2} \alpha}\left(c^{2} \alpha+c^{2}-1\right)}\right. \\
& +\frac{2 d^{2} \Omega_{m o}}{c^{2} \alpha-3 \alpha c^{2} d^{2}-2 c^{2}+2}(1+z)^{3\left(1-d^{2}\right)} \\
& \left.-\frac{c^{2}(1-2 \alpha) \Omega_{c s o}}{2 c^{2} \alpha-c^{2}+1}(1+z)^{6}\right]
\end{aligned}
$$



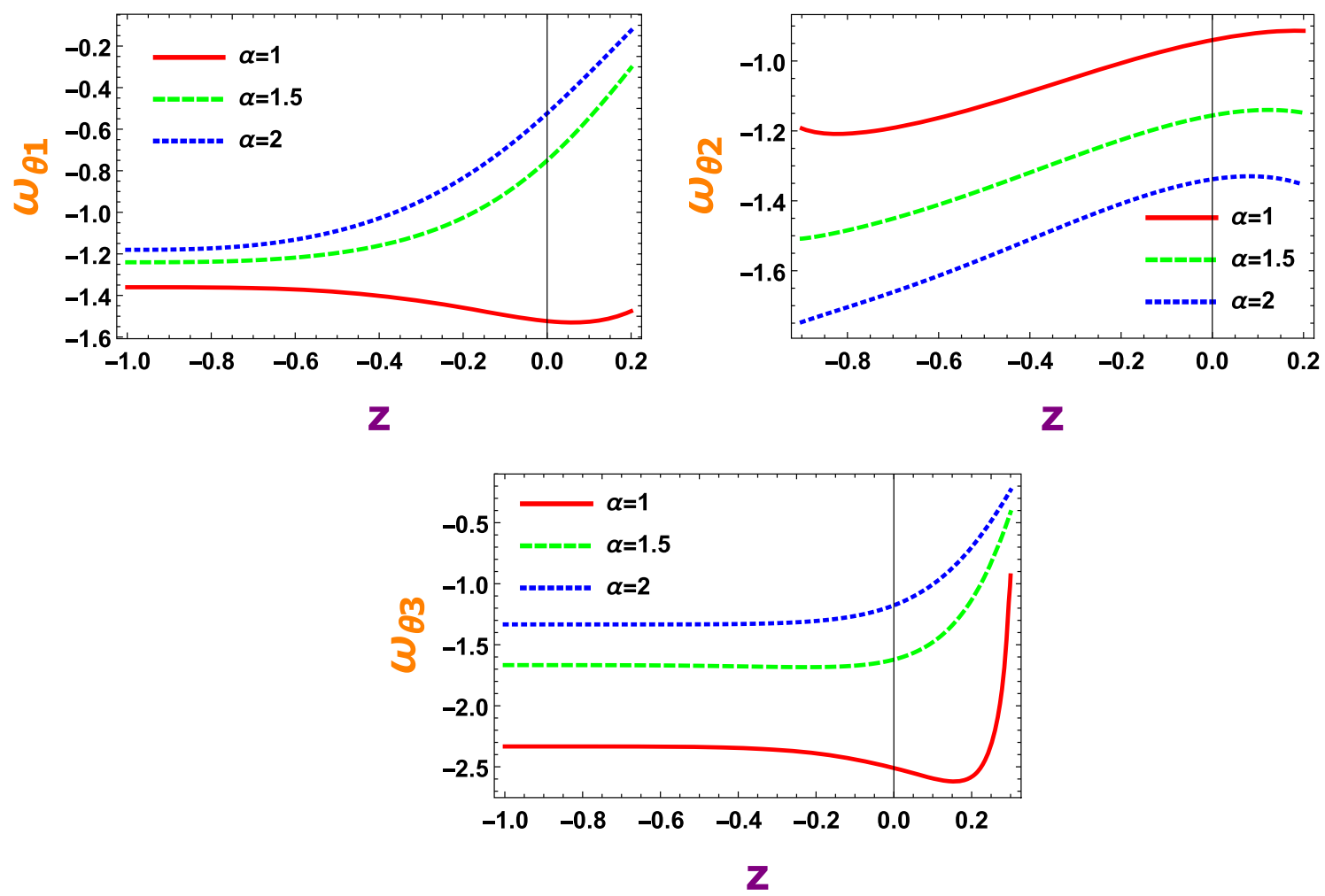

Fig. 3 Plot of EoS parameter $\omega_{1}, \omega_{2}$ and $\omega_{3}$ against redshift $z$

$$
\begin{aligned}
& \times\left[\frac{b}{1+z} e^{\frac{-2}{c^{2} \alpha}\left(c^{2} \alpha+c^{2}-1\right)}\right. \\
& +\frac{c^{2}\left(3 d^{2} \alpha-\alpha+2\right) \Omega_{m o}}{c^{2} \alpha-3 \alpha c^{2} d^{2}-2 c^{2}+2}(1+z)^{3\left(1-d^{2}\right)} \\
& \left.+\frac{c^{2}(1-2 \alpha) \Omega_{c s o}}{2 c^{2} \alpha-c^{2}+1}(1+z)^{6}\right]^{-1}
\end{aligned}
$$

The plot of EoS parameter for model 1 is shown in Fig. 3 (upper left panel) against redshift and GO-HDE $(\alpha)$ parameters. We notice that $\omega_{\theta 1}$ shows accelerated expansion behavior of the universe in three phases such as quintessence, vacuum and phantom. Also, EoS evolutes the universe from quintessence phase to phantom phase by evolving vacuum era. This behavior often called quintom nature of the universe. It is also mentioned here that EoS parameter shows phantom behavior at the present epoch and provides consistence range with Planck observational data for $\alpha=1,1.5$ and 2 [91]. Plot of EoS parameter model 2 is shown in Fig. 3 (upper right panel) against the redshift and $\alpha$ parameters. This represents the quintessence as well as $\Lambda \mathrm{CDM}$ behavior for $\alpha=1,1.5$ and 2. Further, for different parametric values we have compatible behavior of EoS parameter for model 2. It is also observed that the values of EoS parameter remains in suggested ranges of Planck observational data [91]. Plot of EoS parameter for model 3 is shown in Fig. 3 (lower panel) against the redshift and different values of $\alpha$. We notice that $\omega_{\theta 3}$ remains in accelerated expansion eras, i.e., quintessence and vacuum of the universe for specific values of $\alpha$. The values of this parameter is also consistent with observational data.

\subsection{Deceleration parameter}

The deceleration parameter $q$ in terms of $E^{2}$ is written as

$q=-\frac{\ddot{a}}{a H^{2}}=-\left(1+\frac{\dot{H}}{H^{2}}\right)=-1-\frac{1}{2 E^{2}} \frac{d E^{2}}{d x}$.

Using Eqs. (23) and (41), we obtain the deceleration parameter for for model $\mathbf{1}$ as

$$
\begin{aligned}
q_{1}= & -1+\frac{1}{c^{2} \alpha} \\
& {\left[\left(c^{2} \beta-1\right)+\left(\Omega_{m_{0}}(1+z)^{3\left(1-d^{2}\right)}\right.\right.} \\
& \left.+\Omega_{c s_{0}}(1+z)^{6}\right) \times b(1+z)^{\frac{2}{c^{2} \alpha}\left(c^{2} \beta-1\right)} \\
& +\frac{2}{c^{2} \alpha}\left(\frac{\Omega_{m_{0}}(1+z)^{3\left(1-d^{2}\right)}}{3\left(1-d^{2}\right)-\frac{2}{c^{2} \alpha}\left(c^{2} \beta-1\right)}\right. \\
& \left.\left.+\frac{\Omega_{c s_{0}}(1+z)^{6}}{6-\frac{2}{c^{2} \alpha}\left(c^{2} \beta-1\right)}\right)\right]^{-1} .
\end{aligned}
$$



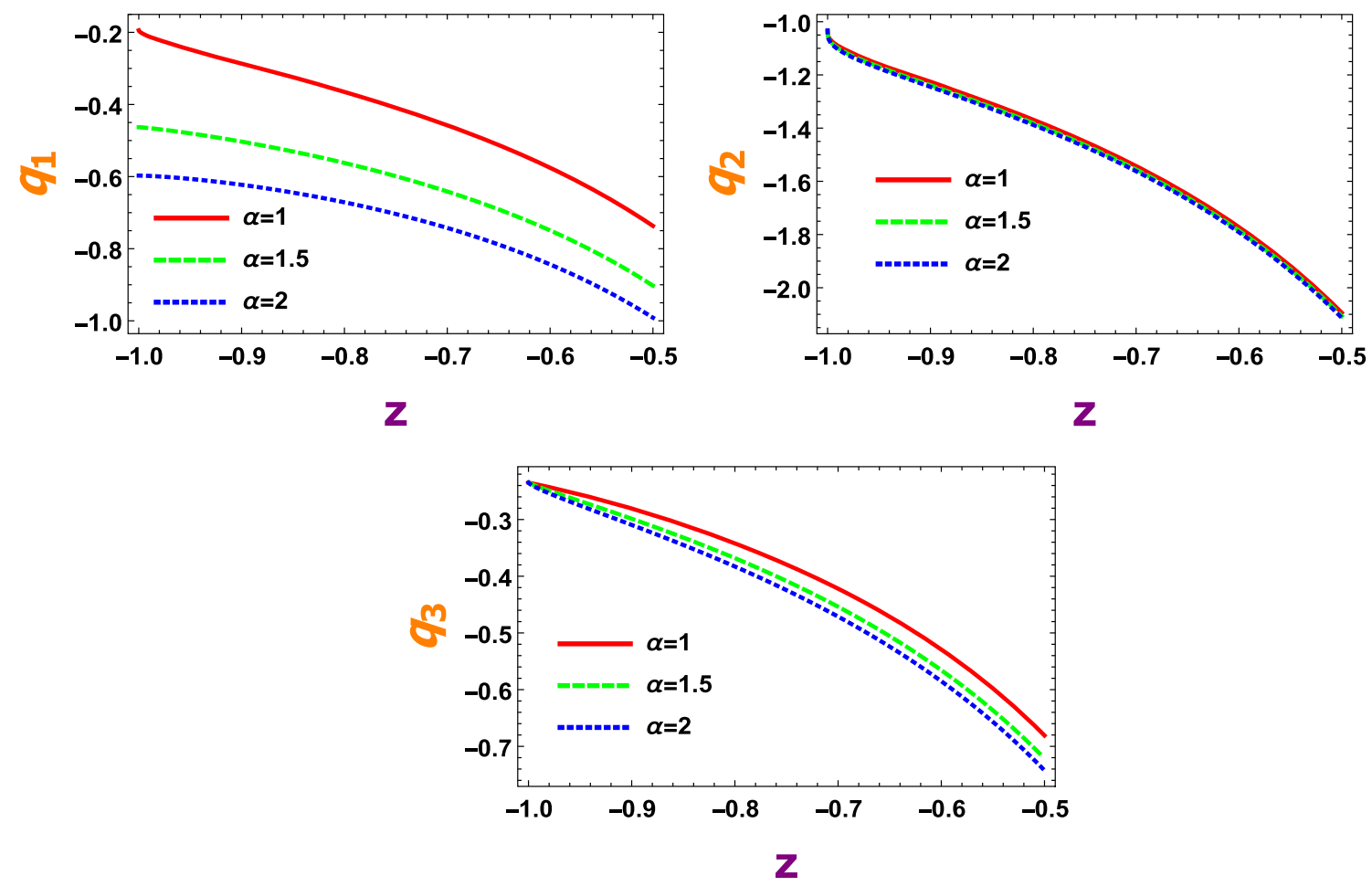

Fig. 4 Plot of deceleration parameter $q_{1}, q_{2}$ and $q_{3}$ versus redshift $z$

For model 2:, one can get deceleration parameter by using Eqs. (26) and (41) as

$$
\begin{aligned}
& q_{2}=-1+\left[\frac { 1 } { 4 \alpha } \left(\frac{b_{1}}{1+z}\left(\beta+\sqrt{\beta^{2}+8 \alpha-8 \alpha \zeta}\right)\right.\right. \\
& \times e^{\frac{-1}{2 \alpha}\left(\beta+\sqrt{\left.\beta^{2}+8 \alpha-8 \alpha \zeta\right)}\right.}+\frac{b_{2}}{1+z} \\
& \left.\times\left(\beta-\sqrt{\beta^{2}+8 \alpha-8 \alpha \zeta}\right) \times e^{\frac{-1}{2 \alpha}\left(\beta-\sqrt{\beta^{2}+8 \alpha-8 \alpha \zeta}\right)}\right) \\
& -\left(3\left(1-d^{2}\right) \Omega_{m_{0}}\right) \\
& \times(1+z)^{3\left(1-d^{2}\right)} \\
& \left(9 \alpha d^{4}+3 \beta d^{2}-18 \alpha d^{2}+9 \alpha-3 \beta+2 \zeta-2\right)^{-1} \\
& -\left(6 \Omega_{c s_{0}}(1+z)^{6}\right) \\
& \left.\times(36 \alpha-6 \beta+2 \zeta-2)^{-1}\right] \\
& \times\left[\frac{b_{1}}{1+z} e^{\frac{-1}{2 \alpha}\left(\beta+\sqrt{\beta^{2}+8 \alpha-8 \alpha \zeta}\right)}+b_{2} e^{\frac{-1}{2 \alpha}\left(\beta-\sqrt{\beta^{2}+8 \alpha-8 \alpha \zeta}\right)}\right. \\
& -\frac{2 \Omega_{m_{0}} \times(1+z)^{3\left(1-d^{2}\right)}}{9 \alpha d^{4}+3 \beta d^{2}-18 \alpha d^{2}+9 \alpha-3 \beta+2 \zeta-2} \\
& -2 \times \Omega_{c s_{0}}(1+z)^{6} \\
& \left.\times(36 \alpha-6 \beta+2 \zeta-2)^{-1}\right]^{-1} \text {. }
\end{aligned}
$$

By using Eqs. (31) and (41), we can obtain the deceleration parameter for model 3 as follows

$$
\begin{aligned}
q_{3}= & \frac{1}{c^{2} \alpha}\left[\left(c^{2} \alpha+c^{2}-1\right)+\left(\Omega_{m_{0}}(1+z)^{3\left(1-d^{2}\right)}\right.\right. \\
& \left.+\Omega_{c s_{0}}(1+z)^{6}\right) \times \frac{b}{1+z} e^{\frac{-2}{c^{2} \alpha}\left(c^{2} \alpha+c^{2}-1\right)} \\
& +\frac{2}{c^{2} \alpha}\left(\frac{\Omega_{m_{0}}(1+z)^{3\left(1-d^{2}\right)}}{3\left(1-d^{2}\right)-\frac{2}{c^{2} \alpha}\left(c^{2} \alpha+c^{2}-1\right)}\right. \\
& +\left(6-\frac{2}{c^{2} \alpha}\left(c^{2} \alpha+c^{2}-1\right)\right)^{-1} \\
& \left.\left.\times \Omega_{c s_{0}}(1+z)^{6}\right)\right]^{-1}-1
\end{aligned}
$$

In Fig. 4, we plot deceleration parameter $q$ versus red-shift parameter $z$ and $\alpha$ for three different HDE models with the assumption of same constant parameters. It is noted that $q$ remains less than zero and also its range lies within suggested range of observational for all three models of HDE [91] for all three models of DE.

\subsection{Stability analysis}

For cosmological models, squared speed sound $v_{s}^{2}$ is used to check their stability. If $v_{s}^{2}<0$ represents instability and $v_{s}^{2}>0$ represents stability. $v_{s}^{2}$ is the ratio of the derivative of pressure and density and can be calculated as

$v_{s j}^{2}=\frac{d p_{j}}{d \rho_{\theta j}}=\frac{\dot{p}_{j}}{\dot{\rho}_{\theta j}}$. 


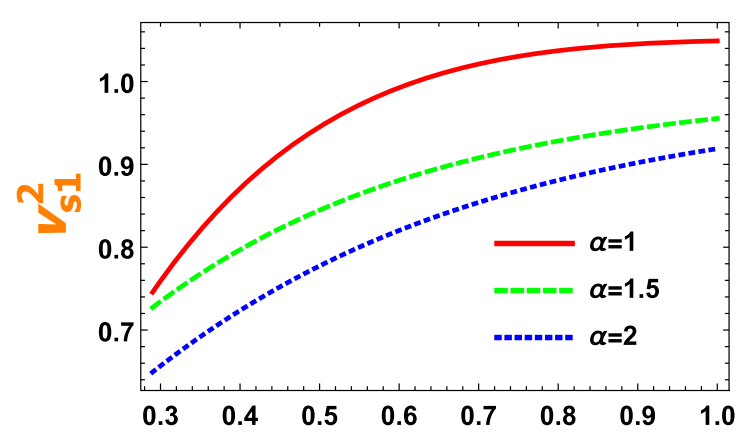

Z

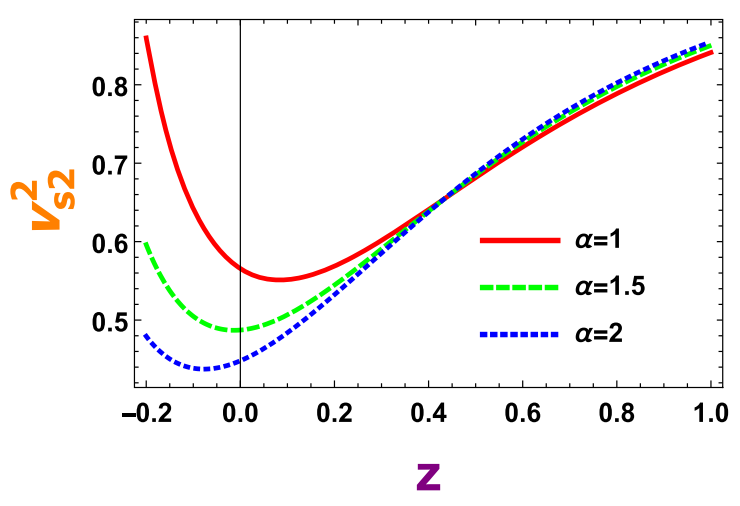

$\mathbf{Z}$

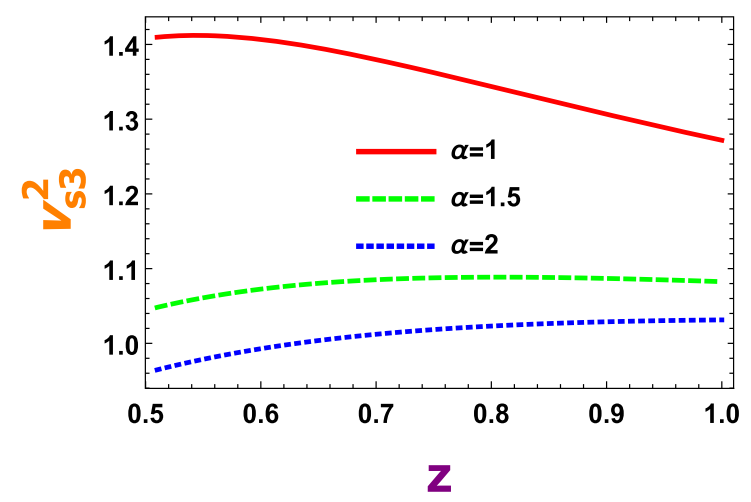

Fig. 5 The plot of square speed of sound $v_{s 1}^{2}, v_{s 2}^{2}$ and $v_{s 3}^{2}$ against redshift $z$

Its working methodology depends upon the density of the perturbation theory from the theory of linear perturbation [92]. Here, we discuss the stability of three different DE models by using cosmological parameter which is called squared speed of sound $\left(v_{s}^{2}\right)$. First of all, we will evaluate the expression of squared sound speed for GO cut-off HDE (model 1) by using Eq. ((19), (22) and Eq. (23)) as

$$
\begin{aligned}
v_{s 1}^{2}= & -\left[\frac{1}{3}\left(-2\left(c^{2} \alpha\right)^{-1}\left(c^{2} \beta-1\right) x\right)\right. \\
& \left(\frac{-2 \beta}{\alpha}+\frac{2}{c^{2} \alpha}+3\right) b(1+z)^{\frac{2}{c^{2} \alpha}}\left(c^{2} \beta-1\right) \\
& -\left(6 d^{2}\left(1-d^{2}\right) \times \Omega_{m o}(1+z)^{3\left(1-d^{2}\right)}\right) \\
& \times\left(3 c^{2} \alpha-3 \alpha c^{2} d^{2}-2 \beta c^{2}+2\right)^{-1} \\
& \left.+\left(6 c^{2}(\beta-3 \alpha) \times \Omega_{c s o}(1+z)^{6}\right)\left(3 c^{2} \alpha-c^{2} \beta+1\right)^{-1}\right] \\
& {\left[\frac{-2}{c^{2} \alpha}\left(c^{2} \beta-1\right) b(1+z) \frac{2}{c^{2} \alpha}\left(c^{2} \beta-1\right)\right.} \\
& -3\left(1-d^{2}\right) \frac{c^{2}\left(2 \beta-3 \alpha+3 \alpha d^{2}\right) \Omega_{m o}}{3 c^{2} \alpha-3 \alpha c^{2} d^{2}-2 \beta c^{2}+2} \\
& (1+z)^{3\left(1-d^{2}\right)}-\frac{6 c^{2}(\beta-3 \alpha)}{3 c^{2} \alpha-c^{2} \beta+1} \\
& \left.\times \Omega_{c s o} \times(1+z)^{6}\right]^{-1} .
\end{aligned}
$$

For model 2, the equation of squared speed of sound leads to

$$
\begin{aligned}
v_{s 2}^{2}= & -\left[\left(-\left(6 \alpha-\beta-\sqrt{\beta^{2}+8 \alpha-8 \alpha \zeta}\right)\left(\beta+\sqrt{\beta}^{2}\right.\right.\right. \\
& +8 \alpha-8 \alpha \zeta))\left(12 \alpha^{2}\right)^{-1} \\
& \times \frac{b_{1}}{1+z} e^{\frac{-1}{2 \alpha}\left(\beta+\sqrt{\beta^{2}+8 \alpha-8 \alpha \zeta}\right)} \\
& -\frac{\left(6 \alpha-\beta+\sqrt{\beta^{2}+8 \alpha-8 \alpha \zeta}\right)\left(\beta-\sqrt{\beta^{2}+8 \alpha-8 \alpha \zeta}\right)}{12 \alpha^{2}} \\
& \times \frac{b_{2}}{1+z} e^{\frac{-1}{2 \alpha}\left(\beta-\sqrt{\beta^{2}+8 \alpha-8 \alpha \zeta}\right)} \\
& +\frac{6\left(1-d^{2}\right) d^{2} \Omega_{m_{0}}(1+z)^{3\left(1-d^{2}\right)}}{9 \alpha d^{4}+3 \beta d^{2}-18 \alpha d^{2}+9 \alpha-3 \beta+2 \zeta-2} \\
& \left.-\frac{6(36 \alpha-6 \beta+2 \zeta) \Omega_{c s_{0}}}{36 \alpha-6 \beta+2 \zeta-2}(1+z)^{6}\right] \\
& \times\left[-(2 \alpha)^{-1} \times\left(\beta+\sqrt{\left.\beta^{2}+8 \alpha-8 \alpha \zeta\right)} \frac{b_{1}}{1+z}\right.\right. \\
\times & e^{\frac{-1}{2 \alpha}\left(\beta+\sqrt{\beta^{2}+8 \alpha-8 \alpha \zeta}\right)} \\
& +\frac{-1}{2 \alpha}\left(\beta-\sqrt{\left.\beta^{2}+8 \alpha-8 \alpha \zeta\right)} b_{2} e^{\frac{-1}{2 \alpha}\left(\beta-\sqrt{\left.\beta^{2}+8 \alpha-8 \alpha \zeta\right)}\right.}\right. \\
& +3\left(1-d^{2}\right) \\
& \frac{9 \alpha d^{4}+3 \beta d^{2}-18 \alpha d^{2}+9 \alpha-3 \beta+2 \zeta \Omega_{m} O}{9 \alpha d^{4}+3 \beta d^{2}-18 \alpha d^{2}+9 \alpha-3 \beta+2 \zeta-2}(1+z)^{3\left(1-d^{2}\right)} \\
& \left.+\frac{6(36 \alpha-6 \beta+2 \zeta) \Omega_{c s} O}{36 \alpha-6 \beta+2 \zeta-2}(1+z)^{6}\right]^{-1} .
\end{aligned}
$$


For model 3, the squared speed of sound equation turns out to be

$$
\begin{aligned}
v_{s 3}^{2}= & -\left[-2\left(3 c^{2} \alpha\right)^{-1}\left(c^{2} \alpha+c^{2}-1\right)\left(\frac{2}{c^{2} \alpha}+1-\frac{2}{\alpha}\right)\right. \\
& \frac{b}{1+z} e^{\frac{-2}{c^{2} \alpha}\left(c^{2} \alpha+c^{2}-1\right)} \\
& -\left(6 d^{2}\left(1-d^{2}\right)\right)\left(c^{2} \alpha-3 \alpha c^{2} d^{2}-2 c^{2}+2\right)^{-1} \Omega_{m o} \\
& \left.\times(1+z)^{3\left(1-d^{2}\right)}+\frac{6 c^{2}(1-2 \alpha) \Omega_{c s o}}{2 c^{2} \alpha-c^{2}+1}(1+z)^{6}\right] \\
& {\left[-2\left(c^{2} \alpha\right)^{-1}\left(c^{2} \alpha+c^{2}-1\right) \frac{b}{1+z}\right.} \\
& \times e^{\frac{-2}{c^{2} \alpha}\left(c^{2} \alpha+c^{2}-1\right)} \\
& -3\left(1-d^{2}\right) \frac{c^{2}\left(3 d^{2} \alpha-\alpha+2\right) \Omega_{m o}}{c^{2} \alpha-3 \alpha c^{2} d^{2}-2 c^{2}+2}(1+z)^{3\left(1-d^{2}\right)} \\
& -6 c^{2}(1-2 \alpha) \Omega_{c s o} \times(1+z)^{6} \\
& \left.\times\left(2 c^{2} \alpha-c^{2}+1\right)^{-1}\right]^{-1} .
\end{aligned}
$$

The plot of squared speed of sound for model $\mathbf{1}$ is shown in Fig. 5 (upper left panel). We see that $\left(v_{s 1}^{2}\right)$ remains positive for $0.3<z<1$ which shows the stability of HDE with GO cut-off. The plot of squared speed of sound for model 2 is shown in Fig. 5 (upper right panel). It can be noted that $v_{s 2}^{2}$ remains positive which shows stable region the model 2 at present, past and future epoch. The plot of speed of sound is shown in Fig. 5 (lower panel). We see that $\left(v_{s 3}^{2}\right)$ remains positive at later epoch for specific range of $\alpha$ which shows stability of the model 3 throughout history of the universe.

\section{Conclusion}

In this section, we have analyzed the behavior of three distinct HDE models and extract the growth factors, growth matter index, fractional DE densities, EoS, deceleration and squared speed sound parameters. We are summarizing the obtained results as follows:

It has been observed that the growth factor indicates the positive behavior with respect to redshift parameter and lies within observational suggested ranges [89] (references therein) for all three models of HDE (Fig. 1). For growth index parameter, we have obtained observationally compatible range i.e. $0<\gamma<1$ [87-89] and different behavior from $\Lambda$ CDM model. for all three models (Fig. 2).

For all three models, EoS parameter has remained in the accelerated expansion era of the universe. Also, we have obtained the Planck observationally ranges of this parameter for all three models [91] (Fig. 3). This model also provided quintom behavior of the universe. As compared to [76], we have obtained consistent result for GO-HDE model as shown in Fig. 3 as transition from decelerated to accelerated phase with respect to $z$. In Fig. 4, we have noted that $q$ remains less than zero and also its range lies within suggested range of observational for all three models of HDE [91]. The squared speed of sound has also investigated which shows stable behavior for all three models (Fig. 5). In [76], the squared speed of sound observed negative behavior as well. However, we have obtained stability of the model for all chosen values. Finally, it is mentioned here that we have found consistent results by choosing value of $c$ observationally.

Data Availability Statement This manuscript has no associated data or the data will not be deposited. [Authors' comment: This is a theoretical study and no experimental data have been listed.]

Open Access This article is licensed under a Creative Commons Attribution 4.0 International License, which permits use, sharing, adaptation, distribution and reproduction in any medium or format, as long as you give appropriate credit to the original author(s) and the source, provide a link to the Creative Commons licence, and indicate if changes were made. The images or other third party material in this article are included in the article's Creative Commons licence, unless indicated otherwise in a credit line to the material. If material is not included in the article's Creative Commons licence and your intended use is not permitted by statutory regulation or exceeds the permitted use, you will need to obtain permission directly from the copyright holder. To view a copy of this licence, visit http://creativecomm ons.org/licenses/by/4.0/.

Funded by $\mathrm{SCOAP}^{3}$.

\section{References}

1. Y.-Y. Keum, Mod. Phys. Lett. A 22, 2131-2142 (2007)

2. Z.-Y. Huang et al., JCAP 0605, 013 (2006)

3. R.R. Caldwell, M. Doran, Phys. Rev. D 69, 103517 (2004)

4. C. Fedeli, arXiv:0812.1097 [astro-ph]

5. S.F. Daniel, Phys. Rev. D 77, 103513 (2008)

6. S.J. Perlmutter et al., Astrophys. J. 517, 565 (1999)

7. A.G. Riess et al., Astron. J. 116, 1009 (1998)

8. T. Koivisto, D.F. Mota, Phys. Rev. D 73, 083502 (2006)

9. S. Oli, Indian J. Phys. 86, 755 (2012)

10. S. Chattopadhyay, A. Pasqua, Indian J. Phys. 87, 1053 (2013)

11. R. Jackiw, S.Y. Pi, Phys. Rev. D 68, 104012 (2003)

12. V. Taveras, N. Yunes, Phys. Rev. D 78, 064070 (2008)

13. G. Calcagni, S. Mercuri, Phys. Rev. D 79, 084004 (2009)

14. S. Mercuri, V. Taveras, Phys. Rev. D 80, 104007 (2009)

15. R. da Rocha, J.G. Pereira, Int. J. Mod. Phys. D 16, 1653 (2007)

16. S. Alexander, N. Yunes, Phys. Rep. 480, 155 (2009)

17. D. Grumiller, N. Yunes, Phys. Rev. D. 77, 044015 (2008)

18. P. Canizares, J.R. Gair, C.F. Sopuerta, Phys. Rev. D. 86, 044010 (2012)

19. K. Bamba et al., Astrophys. Space Sci. 342, 155 (2012)

20. L. Susskind, J. Math. Phys. 36, 6377 (1995)

21. A.G. Cohen et al., Effective field theory, black holes, and the cosmological constant. Phys. Rev. Lett. 82, 4971-4974 (1999)

22. S.D.H. Hsu, Phys. Lett. B 594, 13 (2004)

23. M. Li, Phys. Lett. B 603, 1 (2004)

24. Q.G. Huang, M. Li, JCAP 04, 013 (2004)

25. M. Jamil et al., Phys. Lett. B 679, 172 (2009)

26. R.G. Cai, Phys. Lett. B 657, 228 (2007)

27. C. Gao et al., Phys. Rev. D 79, 043511 (2009)

28. L. Granda, A. Oliveros, Phys. Lett. B 669, 275 (2008) 
29. S. Chen, J. Jing, Phys. Lett. B 679, 144 (2009)

30. R.G. Cai, B. Hu, Y. Zhang, Commun. Theor. Phys. 51, 954 (2009)

31. L. Xu, J. Lu, W. Li, Eur. Phys. J. C 64, 89 (2009)

32. J. Lu et al., Eur. Phys. J. C 71, 1800 (2011)

33. S. Nojiri, S.D. Odintsov, Gen. Relativ. Gravit. 38, 1285 (2006)

34. L. Granda, A. Oliveros, Phys. Lett. B 671, 199 (2009)

35. A. Sheykhi, Phys. Rev. D 84, 107302 (2011)

36. M. Sharif, A. Jawad, Eur. Phys. J. C 72, 2097 (2012)

37. M. Sharif, A. Jawad, Indian J. Phys. 88, 529-539 (2014)

38. E.N. Saridakis, P.F. Gonzalez-Diaz, C.L. Siguenza, Class. Quantum Gravity 26, 165003 (2009)

39. E.N. Saridakis, Nucl. Phys. B 819, 116 (2009)

40. E.N. Saridakis, Phys. Lett. B 660, 138 (2008)

41. E.N. Saridakis, Phys. Lett. B 661, 335 (2008)

42. E.N. Saridakis, Phys. Lett. B 676, 7 (2009)

43. M.R. Setare, E.N. Saridakis, JCAP 0903, 002 (2009)

44. M.R. Setare, E.N. Saridakis, Phys. Lett. B 671, 331 (2009)

45. M.R. Setare, E.N. Saridakis, Phys. Lett. B 671, 331 (2009)

46. A. Jawad, A. Majeed, Astrophy. Space Sci. 356, 375 (2015)

47. A. Jawad, Eur. Phys. J. C 75, 206 (2015)

48. A. Jawad, S. Chattopadhyay, A. Pasqua, Astrophy. Space Sci. 346, $273(2013)$

49. A. Jawad, S. Chattopadhyay, A. Pasqua, Eur. Phys. J. Plus 128, 88 (2013)

50. A. Jawad, S. Chattopadhyay, A. Pasqua, Eur. Phys. J. Plus 129, 54 (2014)

51. A. Jawad, A. Pasqua, S. Chattopadhyay, Astrophy. Space Sci. 344, 489 (2013)

52. A. Jawad, A. Pasqua, S. Chattopadhyay, Eur. Phys. J. Plus 128, 156 (2013)

53. A. Jawad, Astrophy. Space Sci. 353, 691 (2014)

54. A. Jawad, Eur. Phys. J. Plus 129, 207 (2014)

55. K. Bamba, C.Q. Geng, Phys. Lett. B 679, 282 (2009)

56. K. Bamba, C.Q. Geng, S. Tsujikawa, Phys. Lett. B 688, 101 (2010)

57. K. Bamba, C.Q. Geng, S. Nojiri, S.D. Odintsov, EPL 89, 50003 (2010)

58. K. Bamba, C.Q. Geng, JCAP 1006, 014 (2010)

59. K. Bamba, C.Q. Geng, JCAP 1111, 008 (2011)

60. K. Bamba, R. Myrzakulov, S. Nojiri, S.D. Odintsov, Phys. Rev. D 85, 104036 (2012)

61. K. Bamba, M. Jamil, D. Momeni, R. Myrzakulov, Astrophys. Space Sci. 344, 259 (2013)

62. K. Bamba, Int. J. Geom. Methods Mod. Phys. 13, 1630007 (2016)
63. S. Alexander, N. Yunes, Phys. Rep. 480, 1 (2009)

64. P. Horava, D. Minic, Phys. Rev. Lett. 85, 1610 (2000)

65. S.D. Thomas, Phys. Rev. Lett. 89, 081301 (2002)

66. D. Pavon, W. Zimdahl, Phys. Lett. B 628, 206 (2005)

67. W. Zimdahl, D. Pavon, Class. Quantum Gravity 24, 5461 (2007)

68. X. Zhang, Phys. Rev. D 79, 103509 (2009)

69. D.A. Easson, P.H. Frampton, G.F. Smoot, Phys. Lett. B 696, 273 (2011)

70. S. Wang, Y. Wang, M. Li, Phys. Rep. 696, 1 (2017)

71. I.A. Akhlaghi, M. Malekjani, S. Basilakos, H. Haghi, MNRAS 477, $3659(2018)$

72. L.N. Granda, A. Oliveros, Phys. Lett. B 671, 199 (2009)

73. L.N. Granda, A. Oliveros, Phys. Lett. B 669, 275 (2008)

74. M. Li, X.D. Li, S. Wang, Y. Wang, X. Zhang, JCAP 0912, 014 (2009)

75. M. Li, X.D. Li, S. Wang, X. Zhang, JCAP 0906, 036 (2009)

76. A. Jawad, S. Rani, T. Nawaz, Phys. J. Plus 131, 282 (2016)

77. S. Chen, J. Jing, Phys. Lett. B 679, 144 (2009)

78. A. Pasqua, S. Chattopadhyay, Int. J. Theor. Phys. 53, 435 (2014)

79. J. Dunlop et al., Nature 381, 581 (1996)

80. H. Spinrad et al., Astrophys. J. 484, 581 (1999)

81. D. Jain, A. Dev, Phys. Lett. B 633, 436 (2006)

82. M. Kunz, Phys. Rev. D 80, 123001 (2009)

83. M. Baldi et al., MNRAS 440, 75 (2014)

84. E. Berti et al., Class. Quantum Gravity 32, 243001 (2015)

85. A.P. Lightman, P.L. Schechter, Astrophys. J. 74, 831 (1990)

86. L. Wang, P.J. Steinhardt, Astrophys. J. 508, 483 (1998)

87. P.J.E. Peebles, The Large-Scale Structure of the Universe (New Jersey) (Princeton University Press, Princeton, 1980)

88. J.N. Fry, Phys. Lett. B 158, 211 (1985)

89. X. Fu, P. Wu, H. Yu, Int. J. Mod. Phys. D 20, 1301 (2011)

90. Y. Gong, Int. J. Mod. Phys. D 1, 228 (2011)

91. N. Aghanim, Y. Akrami, M. Ashdown et al., Astron. Astrophys. 641, A6 (2020)

92. K.Y. Kim et al., Phys. Lett. B 660, 118 (2008) 